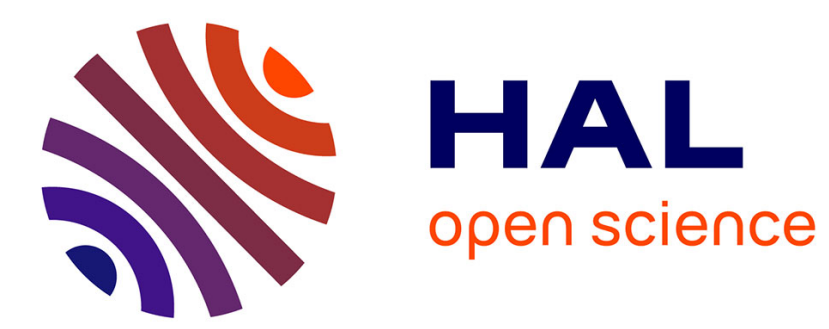

\title{
Reliability of usual assumptions in the calculation of
}

\author{
$\beta$ \\ and \\ $\nu$ \\ spectra \\ Xavier Mougeot
}

HAL Id: hal-01849369

https://hal.science/hal-01849369

Submitted on 9 Jan 2019

HAL is a multi-disciplinary open access archive for the deposit and dissemination of scientific research documents, whether they are published or not. The documents may come from teaching and research institutions in France or abroad, or from public or private research centers.
L'archive ouverte pluridisciplinaire HAL, est destinée au dépôt et à la diffusion de documents scientifiques de niveau recherche, publiés ou non, émanant des établissements d'enseignement et de recherche français ou étrangers, des laboratoires publics ou privés. 


\section{To cite this version:}

Xavier Mougeot. Reliability of usual assumptions in the calculation of

and

$\nu$

spectra. Physical Review C, 2015, 91 (5), pp.055504. 10.1103/PhysRevC.91.055504 . hal-01849369 


\title{
Reliability of usual assumptions in the calculation of $\beta$ and $v$ spectra
}

\author{
X. Mougeot* \\ CEA, LIST, Laboratoire National Henri Becquerel, Gif-sur-Yvette F-91191, France
}

(Received 25 March 2015; published 18 May 2015)

\begin{abstract}
To test the relevance of the most common assumptions in the calculation of beta $(\beta)$ and neutrino $(v)$ spectra, a database of measured shape factors was created, containing 130 distinct transitions. Some analysis parameters were defined to characterize the quality of the calculated spectra. Results from this systematic comparison between theory and experiment are presented in detail. The $\lambda_{k}=1$ approximation for forbidden unique transitions is shown to be systematically worse than the correct calculation of the theoretical shape factors, where the energy dependence of the $\lambda_{k}$ parameters is included. Regarding forbidden nonunique transitions, the $\xi$ approximation is found to be valid for only about half of the first forbidden nonunique transitions and incorrect in all other cases. The criterion $2 \xi / E_{0} \gg 1$ is proved to be insufficient in itself if $2 \xi / E_{0}<100$.
\end{abstract}

\section{INTRODUCTION}

Beta emission properties are crucial features in nuclear data evaluations for building the decay schemes of radionuclides. Regarding $\beta$ spectra, DDEP evaluations [1] as well as NNDC evaluations [2] provide mean energies calculated by the LOGFT program [3]. The other code commonly used for nuclear data is the RADLST program [4]. Both codes use the same assumptions, which are tested in the present work, and can calculate only allowed, first and second forbidden unique transitions. These assumptions are still used in the most recent calculations $[5,6]$.

Obviously, the mean energies depend directly on the shapes of $\beta$ spectra. Furthermore, the energy distribution of the emission probability is needed in ionizing-radiation metrology [7], nuclear medicine for radiotherapy and dosimetry [8], as well as in the nuclear industry for calculations of residual power or post-irradiation fuel management [9]. The measurement of $v$ spectra from reactors, correlated with $\beta$ spectra, is currently developing as a technique of monitoring nuclear facilities and would enable direct access to the amount of fissile material produced for military use [10]. The $\beta$ decays are also known to play an important role in astrophysics, e.g., for the $r$ process, in cosmochronology or for galatic neutrinos [11]. In elementary particle physics, measured $v$ spectra could highlight possible physics beyond the standard model leading to a hypothetical fourth neutrino coupled only to the gravity field [12]. However, a recent study has shed light on the major influence of the uncertainties that can be associated with $\beta$-spectra calculations, casting doubt on the reality of the "reactor neutrino anomaly" [13].

Atomic effects have already been demonstrated in previous work having a substantial influence on the spectrum shape at low $\beta$ energies, which correspond to high $v$ energies [14]. The calculations presented here are less refined. The aim of this work is to clearly point out the usual assumptions that are used in $\beta$ calculations and to test them via a systematic comparison with recommended experimental shape factors. This study

\footnotetext{
*xavier.mougeot@cea.fr
}

is focused on simple and distinct transitions, disregarding measurements where multiple $\beta$ emissions were not isolated or (neutrinoless) double $\beta$ decays. The present calculations of the $\beta$ spectra, performed using Behrens' formalism [15], are set out in detail in Sec. II. Regarding their importance for some research communities, the elements required to calculate the corresponding $v$ spectra are also given, but obviously the comparison with the measurements presented in Sec. III was performed only for $\beta$ spectra.

\section{CALCULATION OF $\beta$ AND v SPECTRA}

Before going into the details of the calculation, some quantities used throughout this work have to be defined. The fine structure constant is denoted by $\alpha$, while $m_{e}$ and $m_{p}$ stand for the electron and proton rest masses, respectively. The values of these constants are from the 2010 CODATA recommended values [16]. The $v$ particles are assumed to be massless in accordance with [15]. Indeed for this study, a nonzero mass less than $0.5 \mathrm{keV}$ would affect very weakly the shape of $\beta$ spectra in the endpoint region, where the emission probability is low. The daughter atomic number is $Z$ and the mass number of the considered isobaric transition is $A$. The nuclear radius $R$ of the daughter atom is given, according to [17], by $R=0.42587 \alpha A^{1 / 3}$, which corresponds to the usual formulation $R=1.2 A^{1 / 3} \mathrm{fm}$. Note that the present calculations are not very sensitive to this value. Other models, as the one given in $[5,6]$, were tested and did not lead to significant changes.

The particle parameters are the following. The kinetic energy of the $\beta$ particle is denoted by $E$. The transition energy $E_{0}$ is the maximum energy of the $\beta$ spectrum. The total energy of the $\beta$ particle including its rest mass is $W=$ $1+E / m_{e}$. Similarly, the total energy corresponding to $E_{0}$ is $W_{0}=1+E_{0} / m_{e}$. The $\beta$-particle momentum is denoted by $p=\sqrt{W^{2}-1}$. Finally, the $\nu$-particle energy is $q=W_{0}-W$, which is also its momentum because of the hypothesis of massless $v$ particles.

Consistent with Behrens' formalism, natural units $\hbar=$ $m_{e}=c=1$ are used throughout this work. All numerical integrations were performed using a local three-point 
Lagrangian interpolation. Each spectrum was normalized to unity.

\section{A. Spectrum shape}

The shape of a $\beta$ spectrum is proportional to (i) a phase space factor $p W q^{2}$ for the sharing of the momentum between the leptons, (ii) the so-called Fermi function $F_{\text {Beh. }}(Z, W)$ which accounts for the static Coulomb corrections from the electromagnetic field of the nucleus, and (iii) a shape factor $C(W)$ which accounts for the remaining energy dependence that comes from the coupling between the nuclear structure and the leptons dynamic. Thus, following Behrens' formalism [15], it is given by

$$
\frac{d N}{d W} \propto p W q^{2} F_{\mathrm{Beh} .}(Z, W) C(W) .
$$

It should be highlighted that Behrens' formalism exhibits a certain symmetry for which the calculation of a $\beta^{-}$decay is performed for a positive $Z$, while a negative $Z$ is used for a $\beta^{+}$ decay. It is also noteworthy that in this formalism $F_{\text {Beh. }}(Z, W)$ is defined differently from the usual Fermi function. Actually, it is the product of the usual Fermi function $F_{0}$ and a factor $L_{0}$ which accounts for the distortion of the electron wave functions by a non-point-like nuclear charge. $F_{0} L_{0}$ can be determined directly from the electron wave functions (see Sec. II B). The theoretical shape factors $C(W)$ and the usual approximations tested in the present work ( $\lambda_{k}=1$ and $\xi$ approximation) are described in Sec. II C. The spectrum given in Eq. (1) is also corrected for the atomic screening effect (Sec. IID) and for radiative corrections (Sec. II E).

The present calculations do not take into account any weak magnetism correction because currently, there are neither theoretical nor experimental bases to establish a clear and trustworthy correction. A recent attempt proposes a first-order correction, but with an associated uncertainty much greater than the corrected effect $[5,6]$. One can expect that a careful study of the experimental shape factors recommended in this work, especially for the allowed and first forbidden unique transitions, could lead to a more precise estimate of the weak magnetism effect. But such a study would require sophisticated calculations that account for the atomic and nuclear structures sufficiently well to clearly deconvolve them.

Because of the conservation of the leptonic charge and of the energy during the transition, there is a symmetry between $\beta$ and $\nu$ spectra, the latter being the complement of the former. Indeed, the probability of a $\beta$ particle to be created with energy $W$ is the same as the probability of a $v$ particle to be created with energy $q=W_{0}-W$ [15]. The only awkward problem concerns the radiative corrections, which are different and lead to a small asymmetry (see Sec. II E).

\section{B. Atomic potential and electron wave functions}

Electron wave functions have to be calculated to determine some parameters $\left(F_{0} L_{0}\right.$ and $\left.\lambda_{k}\right)$ involved in the spectrum shape. Obviously, the structure of the Coulomb potential highly influences these wave functions. A more complicated potential, which was used to calculate precisely the atomic effects in allowed transitions, has already been described elsewhere [14]. Unfortunately, the required computation time for one spectrum is non-negligible and moreover, for most measurements referred to in this work the lowest energies recorded are too high to be sensitive to these atomic effects.

Therefore, the Coulomb potential of a uniformly charged sphere with a nuclear radius $R$ was considered, and it was assumed to be scalar, static, and spherically symmetric. It exhibits a quadratic behavior inside the nucleus and is the same as for a pointlike charge outside,

$$
V(r)= \begin{cases}-\frac{\alpha Z}{2 R}\left[3-\left(\frac{r}{R}\right)^{2}\right] & 0 \leqslant r \leqslant R, \\ -\frac{\alpha Z}{r} & R \leqslant r \leqslant \infty .\end{cases}
$$

The finite nuclear size effect is thus intrinsically included in the present calculations.

The relativistic behavior of the $\beta$ particle within the Coulomb field of the nucleus is described by the Dirac equation. The electron wave function can be split into a radial part and an angular part (see [14] for more details). Thus, it is only necessary to calculate the electron radial wave functions $f_{\kappa}(r)$ (small component) and $g_{\kappa}(r)$ (large component). Here $\kappa$ is the eigenvalue of the operator $\hat{K}=\beta(\vec{\sigma} \cdot \vec{L}+1)$ which appears by applying the theory of angular momentum to an electron in a Coulomb central field ( $\beta$ is the Dirac matrix, $\vec{\sigma}$ designates the Pauli matrices $\sigma_{x, y, z}$, and $\vec{L}$ is the orbital angular momentum operator). Analytical solutions of the Dirac equation for $f_{\kappa}$ and $g_{\kappa}$ exist only for pure Coulomb potential, i.e., $V(r)=-\alpha Z / r$ [18]. The somewhat simple potential in Eq. (2) requires determining the radial wave functions numerically.

Bühring's method was followed, in which the electron radial wave functions are expressed locally as power series expansions, allowing the Dirac equation to be solved with recurrence relations [19]. Specific solutions have to be determined relative to the singular points $r=0$ and $r=\infty$. Behrens and Bühring describe two methods in [15] for determining the global solution. The first one is called "the simple but slow method." The radial wave functions are evaluated (i) near the singular points with their specific solutions and (ii) step by step between these two points using the ordinary solution to (iii) reconnect each solution with the appropriate renormalization and phase shift. The so-called Coulomb amplitudes $\alpha_{\kappa}$ are given, and defined, by the reconnection of the solutions near the origin with the ordinary solutions. These amplitudes are linked to the values of the radial wave functions at the nuclear surface. Similarly, the phase shifts are given by the reconnection of the asymptotic solutions with the ordinary solutions. This procedure is time consuming because it requires evaluating the wave functions in the whole space, without neglecting any spatial oscillation.

The second method, used in this work, is referred to as "the fast but complicated method." Actually, it is possible to avoid evaluating the wave functions in the whole space in the specific case of the potential given in Eq. (2). This method is too complicated to be set out in detail here, however, the basic idea is to choose the solutions relative to the origin as a basis for representing the solution outside the nucleus. The 
reader is urged to look at [15] for a complete explanation. Because of the complexity of this method, validation of the present calculations was attempted here. The unscreened Coulomb functions (Table II in [17]) were recalculated and perfectly reproduced up to the last decimal. Thus, the code implemented for this work can calculate these parameters at any energy, especially the Fermi function $F_{0} L_{0}$ and the $\lambda_{k}$ parameters, avoiding any interpolation in these tables. Indeed in Behrens formalism, the Fermi function includes the finite nuclear size effect through the $L_{0}$ factor and is determined using the Coulomb amplitude of the electron wave functions,

$$
F_{\text {Beh. }}(Z, W)=F_{0} L_{0}=\frac{\alpha_{-1}^{2}+\alpha_{1}^{2}}{2 p^{2}},
$$

disregarding the structure of $V(r)$ and the method used to calculate the wave functions. The $\lambda_{k}$ parameters are defined in Sec. II C.

\section{Theoretical shape factors}

The $\beta$ decays are classified according to the spin change $\Delta J=\left|J_{i}-J_{f}\right|$ and the parity change $\pi_{i} \pi_{f}$, where $\left(J_{i}, \pi_{i}\right)$ and $\left(J_{f}, \pi_{f}\right)$ denote the spins and parities of the initial and final nuclear states, respectively.

For allowed and forbidden unique transitions, Behrens and Bühring have demonstrated that in a first approximation, but with excellent precision, the nuclear current component can be factored out and only the dynamical factor from the coupling with the leptons remains [15]. Introducing $L=1$ for $\Delta J=0$ and $L=\Delta J$ otherwise, the shape factor of an allowed $(L=1)$ or an $(L-1)^{\text {th }}$ forbidden unique transition is

$$
C(W)=(2 L-1) ! \sum_{k=1}^{L} \lambda_{k} \frac{p^{2(k-1)} q^{2(L-k)}}{(2 k-1) ![2(L-k)+1] !},
$$

with $k=|\kappa|$ and $\lambda_{k}(Z, W)$ is determined from the Coulomb amplitudes $\alpha_{\kappa}$ defined in Sec. II B,

$$
\lambda_{k}=\frac{\left(\alpha_{-k}^{2}+\alpha_{k}^{2}\right)}{\left(\alpha_{-1}^{2}+\alpha_{1}^{2}\right)}
$$

As described in Sec. II B, the procedure to calculate these $\lambda_{k}$ parameters is not straightforward. For this reason, a typical, but not justified, assumption in classical $\beta$-spectra calculations, even in the most recent ones $[5,6]$, is to set $\lambda_{k} \equiv 1$. This assumption is referred to as the $\lambda_{k}=1$ approximation in this work.

However, calculation of $\beta$ spectra for nonunique transitions is far more difficult than for unique transitions because the structures of the initial and final nuclear states have to be taken into account. It can be justified for some first forbidden nonunique transitions to calculate them as allowed if the $\xi$ approximation is fulfilled [20], i.e., when the Coulomb energy of the $\beta$ particle emitted at the nuclear surface is much higher than the maximum energy of the transition,

$$
2 \xi=\frac{\alpha Z}{R} \gg E_{0} .
$$

However, despite no theoretical justification, it is often generalized to all nonunique transitions, calculating a forbidden nonunique transition as a forbidden unique one having the same $\Delta J$. No other study was found that evaluates the applicability of the $\xi$ approximation or that sets a limit on the ratio $2 \xi / E_{0}$ for which it would definitely provide good results.

\section{Atomic screening effect}

Screening is the only atomic effect taken into account in this work. Excitation and ionization of atomic electrons, respectively, called shake-up and shake-off effects, can arise from the sudden change of the nucleus charge during the $\beta$ transition. These secondary effects can be confidently set apart because the probability of $K$-shell depletion per $\beta$ decay is expected to be about $0.1 \%$ [21].

Studying the ${ }^{63} \mathrm{Ni}$ and ${ }^{241} \mathrm{Pu}$ decays, it was already proved in previous work that two main atomic effects have to be accounted for to accurately reproduce $\beta$ spectra at low energy, the screening and the exchange effects [14]. A new and precise screening correction was defined at that time for allowed transitions that takes into account the spatial extension of both the potential $V(r)$ and the wave functions, but its calculation is time consuming. In the present work, the common approach described below and originally set out 80 years ago by Rose [22] was preferred to test its influence. This correction is largely found in the literature and still used in modern codes.

In this approach, the screening effect is approximately estimated by subtracting a screened potential of a ThomasFermi type that depends only on $Z$. For $\beta^{-}$transitions, the potential was taken from [23]

$$
\begin{aligned}
V_{0}\left(\beta^{-}\right)= & \left(-9.45 \times 10^{-9}\right)|Z|^{3}+\left(3.014 \times 10^{-6}\right)|Z|^{2} \\
& +\left(1.881 \times 10^{-4}\right)|Z|-\left(5.116 \times 10^{-4}\right) .
\end{aligned}
$$

For $\beta^{+}$transitions, the potential is modified in the following way [24]:

$$
V_{0}\left(\beta^{+}\right)=V_{0}\left(\beta^{-}\right) \exp \left(a / p+b / p^{2}\right),
$$

with the parameters,

$$
\begin{aligned}
a= & \left(1.11 \times 10^{-7}\right)|Z|^{3}-\left(1.01 \times 10^{-5}\right)|Z|^{2} \\
& -\left(2.38 \times 10^{-3}\right)|Z|+0.102,
\end{aligned}
$$

and

$$
\begin{aligned}
b= & \left(-2.42 \times 10^{-8}\right)|Z|^{3}+\left(3.83 \times 10^{-6}\right)|Z|^{2} \\
& +\left(3.60 \times 10^{-5}\right)|Z|-0.0156 .
\end{aligned}
$$

The method for applying this correction is well described in [24] and consists of evaluating the probability of $\beta^{-}$ emission at a given energy $W$ as the same probability as at the energy $W^{\prime}=W-V_{0}$. This can be summarized by a simple three-part rule, where the screening is applied everywhere in the spectrum shape in Eq. (1) and in the subsequent factors in Eqs. (3)-(5) by the following: replacing $W$ by $W^{\prime}$; replacing $p$ by the corresponding momentum $p^{\prime}=\sqrt{W^{\prime 2}-1}$; keeping the $v$ energy $q$ as it is. Obviously, this correction cannot be applied for $W-V_{0}<1$ because $p$ would become imaginary. This proviso induces a nonphysical discontinuity in the spectrum at the energy $E_{\mathrm{sc}}=m_{e} V_{0}$, where the screening correction can 
TABLE I. Experimental shape factors of allowed transitions. Energy range, $\bar{E}_{\text {sf }}$ and $\bar{E}_{\text {calc }}$ are in keV; $\% E_{0},\left(1-R^{2}\right)$ and $\Delta \bar{E}$ are in $\%$.

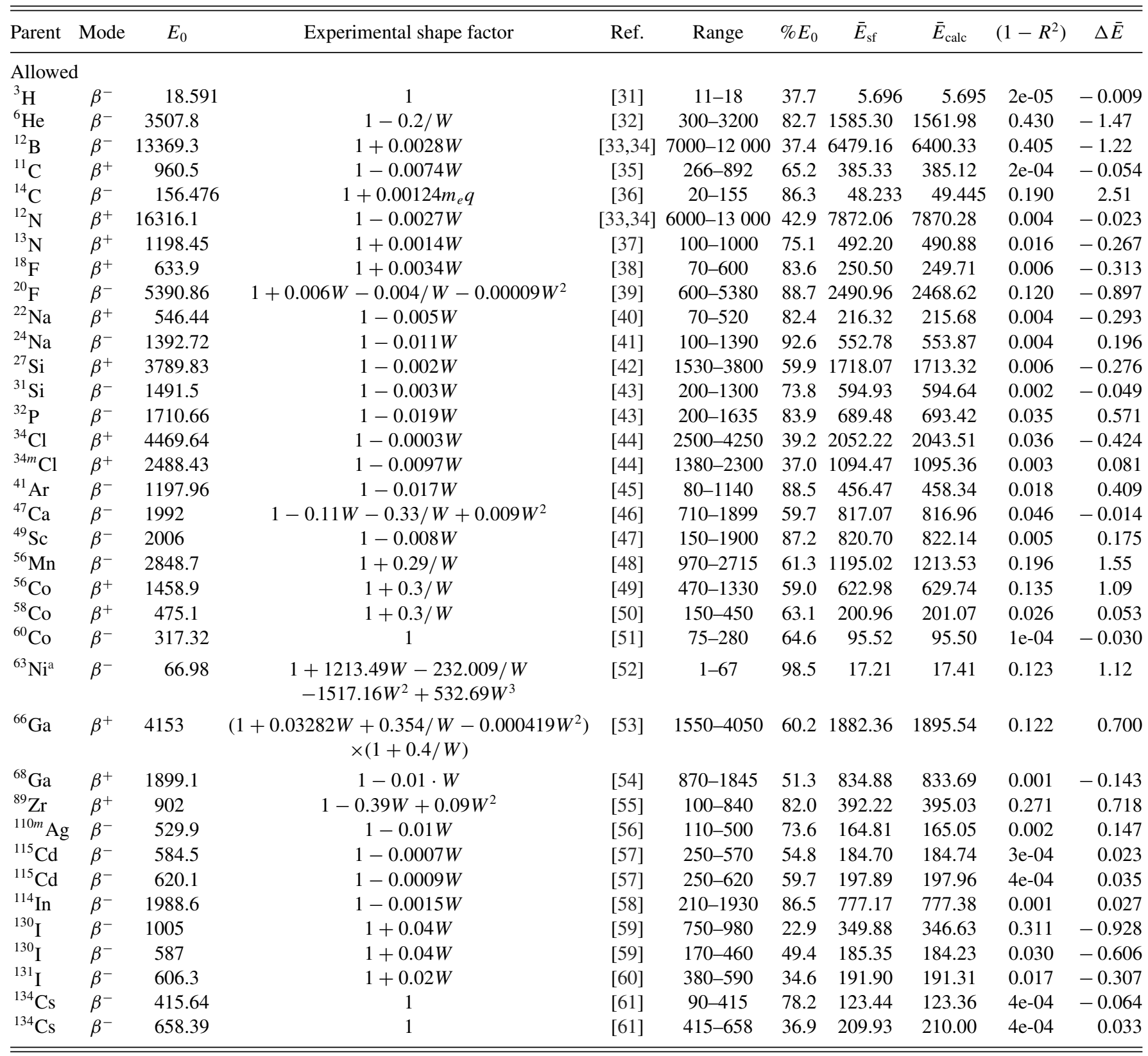

${ }^{\text {a See }}[14]$ for more precise results.

be switched on. Conversely for $\beta^{+}$transitions, the energy $W$ is replaced by $W^{\prime}=W+V_{0}$, the momentum $p$ by the corresponding $p^{\prime}$, and the correction can be applied across the entire energy range of the spectrum.

\section{E. Radiative corrections}

Up to now, only static Coulomb corrections have been considered and atomic effects have been taken into account only through the screening correction, excluding all other atomic processes (see Sec. II D). The radiative corrections are nonstatic Coulomb corrections and they can have an important effect on $\beta$-spectra shapes for high $E_{0}$. Formally, they should be determined using Feynman diagrams, which is an approach far outside the present work. However, they can be split into "inner" and "outer" radiative corrections and only the latter influences the energy dependence of the $\beta$ spectrum [25]. Thus, the "inner" radiative corrections have been left out in present calculations.

Outer radiative corrections account for the energy loss of the $\beta$ particle in the electromagnetic field of the nucleus by means of virtual photons and internal bremsstrahlung. In the present work, they are calculated as described in $[15,25,26]$. The spectrum is corrected by a factor $\left[1+\delta_{R}(W, Z)\right]$ defined as follows:

$$
\delta_{R}(W, Z)=\delta_{1}(W)+\delta_{2}(Z)+\delta_{3}(Z)+\delta_{4}(Z) .
$$

The energy-dependent term is of order $\alpha$,

$$
\delta_{1}(W)=\frac{\alpha}{2 \pi} g(W, q),
$$




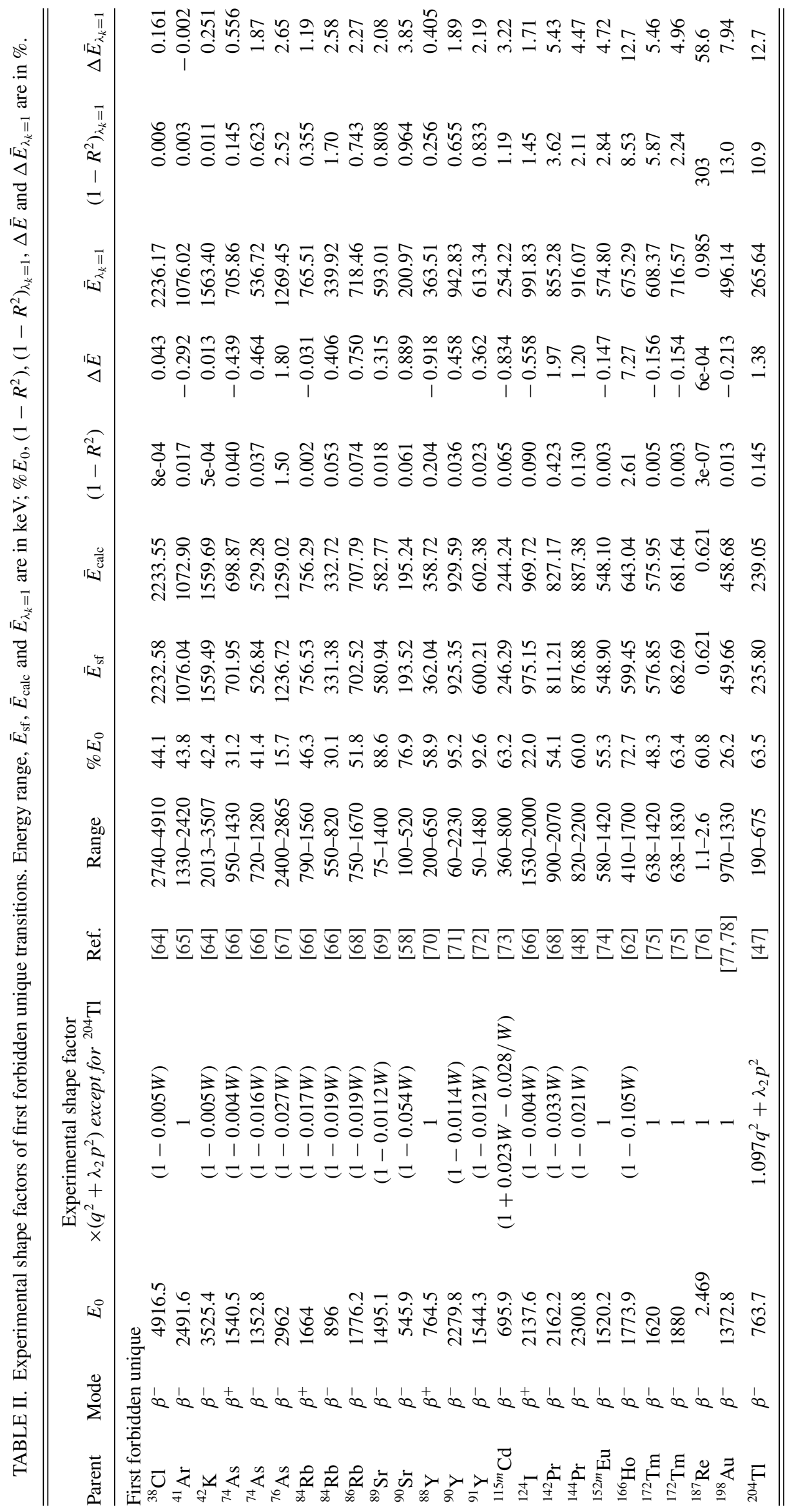


where

$$
\begin{aligned}
g(W, q)= & 3 \ln \left(\frac{m_{p}}{m_{e}}\right)-\frac{3}{4}+\frac{4}{\beta} L\left(\frac{2 \beta}{1+\beta}\right) \\
& +4\left(\frac{\tanh ^{-1} \beta}{\beta}-1\right)\left[\frac{q}{3 W}-\frac{3}{2}+\ln (2 q)\right] \\
& +\frac{\tanh ^{-1} \beta}{\beta}\left[\left(1+\beta^{2}\right) \frac{q^{2}}{3 W^{2}}-4 \tanh ^{-1} \beta\right],
\end{aligned}
$$

with $\beta=p / W$ and $L(x)$ is the Spence function, which is the opposite of the dilogarithm function,

$$
L(x)=\int_{0}^{x} \frac{\ln (1-t)}{t} d t .
$$

The higher order terms in $(\alpha Z)$ are to a first approximation energy independent,

$$
\begin{aligned}
& \delta_{2}(Z)=1.1|Z| \alpha^{2} \frac{m_{p}}{m_{e}}, \\
& \delta_{3}(Z)=\frac{Z^{2} \alpha^{3}}{\pi}\left(3 \ln 2-\frac{3}{2}+\frac{\pi^{2}}{3}\right) \frac{m_{p}}{m_{e}}, \\
& \delta_{4}(Z)=\frac{|Z| \alpha^{3}}{2 \pi} \frac{m_{p}}{m_{e}} .
\end{aligned}
$$

These radiative corrections have recently been calculated for $v$ particles and it was proved that the internal bremsstrahlung contribution is different, the rationale being that nonstatic Coulomb processes are secondary processes [27]. Thus, in present calculations of $v$ spectra, only the following result from [27] is accounted for, which modifies the spectrum by a factor $\left[1+\delta_{v}(q)\right]$ defined as follows:

$$
\delta_{v}(q)=\frac{\alpha}{2 \pi} h(W)
$$

with

$$
\begin{aligned}
h(W)= & 3 \ln \left(\frac{m_{p}}{m_{e}}\right)+\frac{23}{4}+\frac{8}{\beta} L\left(\frac{2 \beta}{1+\beta}\right) \\
& +8\left(\frac{\tanh ^{-1} \beta}{\beta}-1\right) \ln (2 W \beta) \\
& +4 \frac{\tanh ^{-1} \beta}{\beta}\left(\frac{7+3 \beta^{2}}{8}-2 \tanh ^{-1} \beta\right),
\end{aligned}
$$

where $W$ is still the $\beta$-particle energy that corresponds to the $v$ energy $q$, and the quantity $\beta=p / W$ still remains the same.

This difference in the radiative corrections is the only one in the present work that breaks the symmetry between a given $\beta$ spectrum and its corresponding $v$ spectrum.

\section{SYSTEMATIC COMPARISON WITH EXPERIMENTAL SHAPE FACTORS}

A database of $130 \beta$ transitions was created from the experimental shape factors available in the literature. These shape factors come mainly from [28] but with a different choice of the recommended publication in some cases. The literature since [28] was also reviewed and new shape factors have been considered. The vast majority (72\%) were established from measurements using magnetic spectrometers. It is noteworthy that for some transitions, significant disagreement exists between different publications. Each transition energy was updated with the latest DDEP evaluation [29], or with the latest NNDC evaluation [2] if the parent radionuclide was not yet evaluated by the DDEP collaboration.

Calculations have been performed as described in Sec. II to analyze the adequacy of these experimental shape factors. Each spectrum was calculated across the full energy range of the transition. The analysis was handled using parameters defined in Sec. III A and the results are extensively presented in Sec. III B, grouped by the transition type. The calculations have been performed with and without the screening correction presented in Sec. IID. The influence of this correction on the results is weak, especially when $E_{0}$ is high, but no systematic trend was found. Therefore, only results with screening accounted for are given in the present publication.

\section{A. Analysis}

\begin{tabular}{|c|c|c|c|c|c|c|c|c|c|c|c|c|c|}
\hline Parent & Mode & $E_{0}$ & Experimental shape factor & Ref. & Range & $\% E_{0}$ & $\bar{E}_{\mathrm{sf}}$ & $\bar{E}_{\text {calc }}$ & $\left(1-R^{2}\right)$ & $\Delta \bar{E}$ & $\bar{E}_{\lambda_{k}=1}$ & $\left(1-R^{2}\right) \lambda_{k}=1$ & $\Delta \bar{E}_{\lambda_{k}=1}$ \\
\hline \multicolumn{14}{|c|}{ Second forbidden unique } \\
\hline${ }^{10} \mathrm{Be}$ & $\beta^{-}$ & 556 & $q^{4}+(10 / 3) q^{2} p^{2}+p^{4}$ & [79] & $100-500$ & 71.9 & 252.33 & 252.00 & 0.006 & -0.131 & 252.14 & 0.003 & -0.074 \\
\hline${ }^{60} \mathrm{Co}$ & $\beta^{-}$ & 1490.56 & $q^{4}+(10 / 3) \lambda_{2} q^{2} p^{2}+\lambda_{3} p^{4}$ & [81] & $1350-1450$ & 6.7 & 624.50 & 623.47 & 0.073 & -0.166 & 630.94 & 0.060 & 1.03 \\
\hline${ }^{138} \mathrm{La}$ & $\beta^{-}$ & 258 & $\begin{array}{l}1+407.71 W-50.695 / W \\
-583.794 W^{2}+246.279 W^{3}\end{array}$ & [63] & $2.5-255$ & 97.9 & 90.48 & 95.55 & 3.26 & 5.60 & 112.42 & 57.7 & 24.3 \\
\hline \multicolumn{14}{|c|}{ Third forbidden unique } \\
\hline
\end{tabular}

For each $\beta$ transition, the experimental shape factor was not necessarily measured in the full energy range. It is an important feature of the measurement which clearly has a significant influence on the present analysis. Thus, energy range of the measurement and proportion $\left(\% E_{0}\right)$ of this range compared to the maximum available energy $E_{0}$ are provided for each considered transition. This simple criterion highlights that in many cases, the $\beta$ spectra are not so well measured: Forty-four

TABLE III. Experimental shape factors of second and third forbidden unique transitions. Energy range, $\bar{E}_{\text {sf }}, \bar{E}_{\text {calc }}$, and $\bar{E}_{\lambda_{k}=1}$ are in keV; $\% E_{0},\left(1-R^{2}\right),\left(1-R^{2}\right)_{\lambda_{k}=1}, \Delta \bar{E}$, and $\Delta \bar{E}_{\lambda_{k}=1}$ are in $\%$. 
TABLE IV. Experimental shape factors of first forbidden nonunique transitions. Energy range, $\bar{E}_{\mathrm{sf}}$, and $\bar{E}_{\text {calc }}$ are in keV; $\% E_{0},\left(1-R^{2}\right)$, and $\Delta \bar{E}$ are in $\%$.

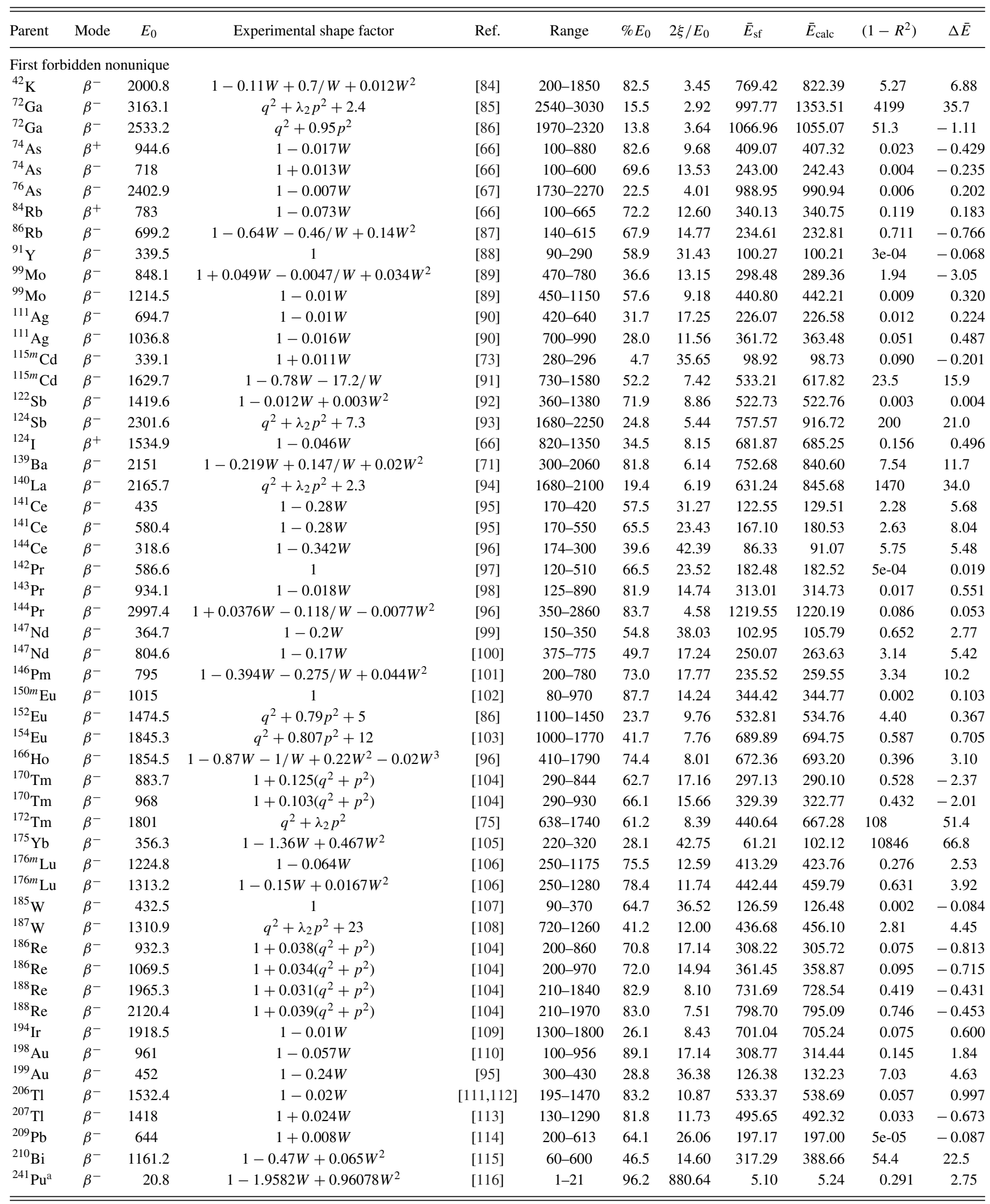

${ }^{\mathrm{a}} \mathrm{See}[14]$ for more precise results. 
transitions over a total of 130 exhibit $\left(\% E_{0}\right)<50 \%$, namely about one-third of the transitions.

The mean energy of each calculated $\beta$ spectrum, from an experimental shape factor or according to one or another assumption, was determined as follows:

$$
\bar{E}=m_{e} \frac{\int_{1}^{W_{0}}(W-1) N(W) d W}{\int_{1}^{W_{0}} N(W) d W} .
$$

The difference $\Delta \bar{E}$ with the mean energy $\bar{E}_{\text {sf }}$ determined from the experimental shape factor is also calculated.

To evaluate the global quality of present calculated spectra, a goodness-of-fit parameter was estimated. Considering a set of $n$ measurements $\left\{y_{i}\right\}$ and a model that leads to $n$ predictions $\left\{y_{i}^{\text {th }}\right\}$, the quality of the fit of this model with respect to the data can be given by

$$
R^{2}=1-\frac{\operatorname{var}\left(\hat{e}_{i}\right)}{\operatorname{var}\left(y_{i}\right)}
$$

This is a general definition which is valid for $n \gg 1$, without any restriction on the type of model. This quantity falls in the range $0 \leqslant R^{2} \leqslant 1$, where $R^{2}=1$ corresponds to a perfect prediction. The modeling error is $\hat{e}_{i}=y_{i}-y_{i}^{\text {th }}$. Nonbiased estimators of the variances are

$\operatorname{var}\left(y_{i}\right)=\sum_{i=1}^{n} \frac{\left(y_{i}-\bar{y}\right)^{2}}{n-1}, \quad \operatorname{var}\left(\hat{e}_{i}\right)=\sum_{i=1}^{n} \frac{\left(\hat{e}_{i}\right)^{2}}{n-N_{p}-1}$,

with $\bar{y}$ the simple mean of the measurements, and $N_{p}$ the number of model parameters. For $\beta$ spectra, $E_{0}$ is clearly one parameter. An additional one is also needed to take into account that the shape factors had been determined from measured spectra, which somewhat hides a normalization. Thus, $N_{p}=2$ was considered to determine $R^{2}$. Therefore, the parameter $\left(1-R^{2}\right)$ quantifies the general disagreement between the measurements and the predictions. For the consistency of the analysis, the $\left(1-R^{2}\right)$ values have only been calculated in the energy range of the measurements.
For forbidden unique transitions, the mean energy $\bar{E}_{\text {calc }}$ refers to the calculated spectrum for which the $\lambda_{k}=1$ approximation was not considered. This approximation was used to calculate the mean energy $\bar{E}_{\lambda_{k}=1}$ and all other parameters having the same label. For forbidden nonunique transitions, the ratio $2 \xi / E_{0}$ is also given because it should determine, in principle, the validity of the $\xi$ approximation. The mean energy $\bar{E}_{\text {calc }}$ and all other parameters then refer to the calculated spectrum according to the $\xi$ approximation, as defined in Sec. II C.

In the following, a $\beta$ spectrum is considered well known in the energy range of the measurement if $\left(1-R^{2}\right)<1 \%$ and $|\Delta \bar{E}|<1 \%$, which will be referred to as the $1 \%$ criterion hereafter. If $\left(1-R^{2}\right)$ is low but $|\Delta \bar{E}|$ is high, it means that the calculation is correct only inside this energy range and, because the mean energy is determined using the full energy range of the transition, then the agreement is significantly worse outside.

\section{B. Results}

The results of this systematic comparison with experimental shape factors are presented first for the allowed transitions in Sec. III B 1. Next, they are shown for the forbidden unique transitions in Sec. III B 2 and the reliability of the $\lambda_{k}=1$ approximation is reviewed. Finally, the results for the forbidden nonunique transitions are given in Sec. III B 3 and a critical point of view on the $\xi$ approximation is provided.

\section{Allowed transitions}

Results for the allowed transitions are presented in Table I. The disagreement parameter $\left(1-R^{2}\right)$ is found to be less than $0.45 \%$ in all cases. With $|\Delta \bar{E}|<1 \%, 30$ of the 36 tabulated spectra are reproduced well. It should be noted that better results were found for the ${ }^{63} \mathrm{Ni}$ decay with more precise calculation of the atomic effects [14]. If this criterion is

TABLE V. Experimental shape factors of second, third, and fourth forbidden nonunique transitions. Energy range, $\bar{E}_{\mathrm{sf}}$, and $\bar{E}_{\text {calc }}$ are in keV;

\begin{tabular}{|c|c|c|c|c|c|c|c|c|c|c|c|}
\hline Parent & Mode & $E_{0}$ & Experimental shape factor & Ref. & Range & $\% E_{0}$ & $2 \xi / E_{0}$ & $\bar{E}_{\text {sf }}$ & $\bar{E}_{\text {calc }}$ & $\left(1-R^{2}\right)$ & $\Delta \bar{E}$ \\
\hline \multicolumn{12}{|c|}{ Second forbidden nonunique } \\
\hline${ }^{36} \mathrm{Cl}$ & $\beta^{-}$ & 709.53 & $\begin{array}{c}1-0.282715 W-0.045988 / W \\
-0.491739 W^{2}+0.438731 W^{3}\end{array}$ & {$[117]$} & $6-710$ & 99.2 & 9.22 & 314.09 & 278.40 & 25.0 & -11.4 \\
\hline${ }^{46} \mathrm{Sc}$ & $\beta^{-}$ & 1477.2 & $q^{2}+1.67 p^{2}$ & {$[81]$} & $1170-1400$ & 15.6 & 4.99 & 657.73 & 603.04 & 19.1 & -8.31 \\
\hline${ }^{59} \mathrm{Fe}$ & $\beta^{-}$ & 1565 & $q^{2}+0.59 p^{2}$ & {$[118]$} & $470-1550$ & 69.0 & 5.32 & 582.92 & 633.75 & 5.54 & 8.72 \\
\hline${ }^{58} \mathrm{Co}$ & $\beta^{+}$ & 1285.9 & $p^{2}$ & [119] & $750-1150$ & 31.1 & 6.27 & 708.71 & 580.91 & 114 & -18.0 \\
\hline${ }^{94} \mathrm{Nb}$ & $\beta^{-}$ & 471.5 & $q^{2}$ & {$[120]$} & $160-450$ & 61.5 & 23.51 & 94.38 & 165.74 & 227 & 75.6 \\
\hline${ }^{99} \mathrm{Tc}$ & $\beta^{-}$ & 293.8 & $q^{2}+0.54 p^{2}$ & {$[121]$} & $55-250$ & 66.4 & 38.84 & 95.28 & 101.03 & 2.72 & 6.04 \\
\hline${ }^{129} \mathrm{I}$ & $\beta^{-}$ & 151.2 & $3.16 q^{2}+p^{2}$ & [122] & $55-145$ & 59.5 & 84.81 & 45.98 & 48.62 & 2.88 & 5.75 \\
\hline${ }^{135} \mathrm{Cs}$ & $\beta^{-}$ & 268.7 & $q^{2}+0.10 p^{2}$ & [123] & $50-195$ & 54.0 & 48.75 & 61.57 & 88.44 & 50.6 & 43.6 \\
\hline${ }^{137} \mathrm{Cs}$ & $\beta^{-}$ & 1175.63 & see reference & [124] & $710-1125$ & 35.3 & 11.09 & 284.90 & 422.87 & 1834 & 48.4 \\
\hline \multicolumn{12}{|c|}{ Third forbidden nonunique } \\
\hline${ }^{87} \mathrm{Rb}$ & $\beta^{-}$ & 283.3 & $q^{4}+0.305 q^{2} p^{2}+0.011 p^{4}$ & {$[125]$} & $65-180$ & 40.6 & 36.32 & 56.65 & 114.99 & 262 & 103 \\
\hline \multicolumn{12}{|c|}{ Fourth forbidden nonunique } \\
\hline${ }^{113} \mathrm{Cd}$ & $\beta^{-}$ & 322 & $\begin{array}{c}3.034 q^{6}+1.499 \times 7 q^{4} p^{2} \\
+1.016 \times 7 q^{2} p^{4}+p^{6}\end{array}$ & {$[126]$} & $30-320$ & 90.1 & 37.77 & 139.83 & 139.30 & 0.076 & -0.376 \\
\hline
\end{tabular}
$\% E_{0},\left(1-R^{2}\right)$, and $\Delta \bar{E}$ are in $\%$. 
extended to $|\Delta \bar{E}|<2 \%, 35$ transitions can be considered as well known.

Only the ${ }^{14} \mathrm{C}$ decay remains for which $|\Delta \bar{E}|=2.51 \%$, which does not fail so badly. This allowed transition exhibits an anomalously long half-life, which clearly suggests a nuclear structure effect which would also affect the spectral shape [30].

\section{Forbidden unique transitions}

In Table II are presented the results for the first forbidden unique transitions, and in Table III for the second and third forbidden unique ones.

Using the $1 \%$ criterion defined in Sec. III A, 21 of the 25 first forbidden unique spectra are reproduced well. Extending the disagreement parameter to $\left(1-R^{2}\right)<2 \%$ and the mean energy parameter to $|\Delta \bar{E}|<2 \%, 24$ transitions can be seen as well known. The only one for which the calculation really fails is the ${ }^{166}$ Ho decay. Despite the measured energy covers $72 \%$ of $E_{0}$ and an emission probability of $50 \%$, the quality of this measurement is perhaps questionable because the authors did not provide much detail of possible source impurities or the data analysis [62]. A new precise measurement would be beneficial.

It is difficult to be conclusive regarding the second and third forbidden unique transitions because of the very small number of measurements. With the $1 \%$ criterion, three of the four second forbidden unique spectra are reproduced well. The last one is the ${ }^{138}$ La decay which was very precisely measured in recent work down to $2.5 \mathrm{keV}$ and for which atomic effects are expected to play an important role [63]. The third forbidden unique transition from the ${ }^{40} \mathrm{~K}$ decay exhibits a disagreement parameter $\left(1-R^{2}\right)=1.21 \%$ and $|\Delta \bar{E}|=0.122 \%$. As it is the only measured transition of this type, it cannot be established if the present calculations are correct for this type of transition in general.

From this analysis, it was found that for all the 30 forbidden unique transitions, results from the $\lambda_{k}=1$ assumption are systematically worse than the full calculation with the correct $\lambda_{k}$ except for the first forbidden unique decay of ${ }^{41} \mathrm{Ar}$ and the second forbidden unique decays of ${ }^{22} \mathrm{Na}$ and ${ }^{10} \mathrm{Be}$. In all three transitions the results pass the $1 \%$ criterion. Thus, these isolated cases cannot be rationally considered as significant regarding the relevance of the $\lambda_{k}=1$ approximation.

\section{Forbidden nonunique transitions}

Results are shown in Table IV for the first forbidden nonunique decays, and in Table $\mathrm{V}$ for the second, third, and fourth forbidden nonunique ones. Analysis parameters $\left(1-R^{2}\right)$ and $|\Delta \bar{E}|$ are presented in Fig. 1 as a function of $2 \xi / E_{0}$.

Applying the $1 \%$ criterion, 26 of the 53 first forbidden nonunique transitions are reproduced well, namely $49 \%$. For the ${ }^{241} \mathrm{Pu}$ decay, better results were found with a more precise calculation of the atomic effects [14]. None of the second and third forbidden nonunique transitions are well reproduced, but the fourth one from the ${ }^{113} \mathrm{Cd}$ decay passes the $1 \%$ criterion. However, no clear statement about the validity of the $\xi$ approximation for this transition type can be made from this single measurement.

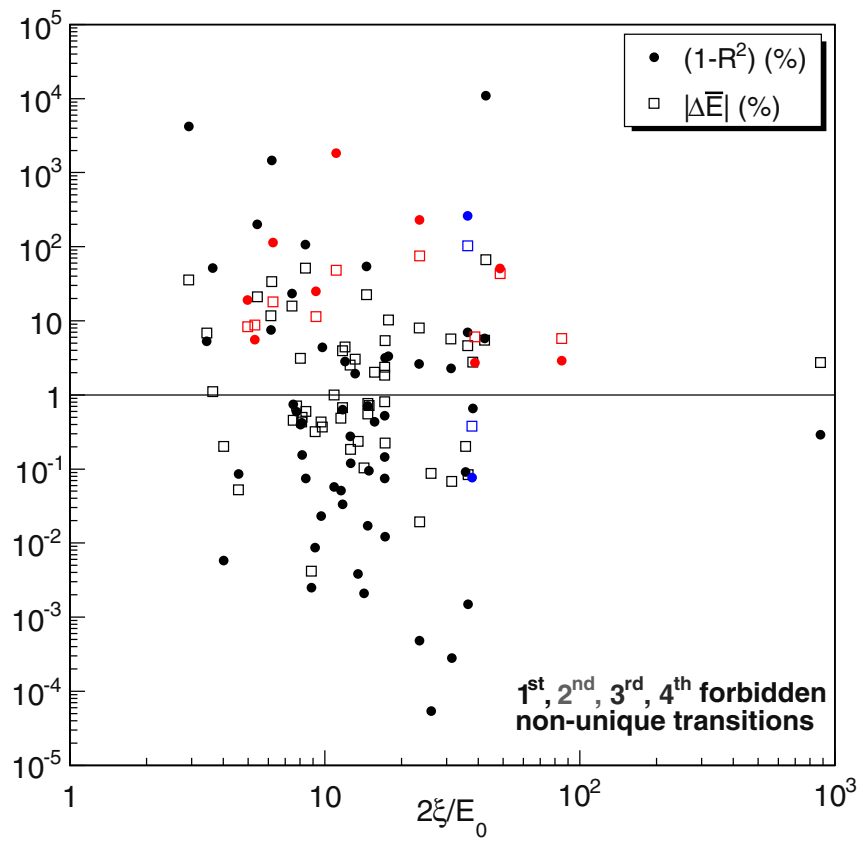

FIG. 1. (Color online) Distribution according to $2 \xi / E_{0}$ of the $\left(1-R^{2}\right)$ and $|\Delta \bar{E}|$ parameters for the measured shape factors of forbidden nonunique transitions.

It is difficult to be conclusive regarding the $\xi$ approximation for extricating a correct criterion. Indeed, one can see in Fig. 1 and in Tables IV and V that the criterion $2 \xi / E_{0} \gg 1$ is not sufficient to predict the correctness of the calculations. A conservative criterion could be $2 \xi / E_{0}>100$, which is confirmed by the first forbidden nonunique decay of ${ }^{151} \mathrm{Sm}$, not present in the tables because no shape factor was yet extracted. However, this transition, with $E_{0}=76.6 \mathrm{keV}$ and $2 \xi / E_{0}=204$, was recently measured and proved to exhibit an allowed shape [83]. Below $2 \xi / E_{0}=100$, no systematic trend can be observed.

\section{CONCLUSION}

Setting out in detail the $\beta$ and $v$ calculations used in this work, the usual assumptions have been clearly pointed out. A database of measured shape factors from distinct $\beta$ emissions was created. The usual assumptions were tested by comparison with the measurements and focus was made on the $\lambda_{k}=1$ approximation for the forbidden unique transitions and the $\xi$ approximation for the forbidden nonunique ones. The main conclusions that can be drawn from this work are the following:

(1) For allowed transitions, except in the specific case of the ${ }^{14} \mathrm{C}$ decay, excellent agreement was found between theory and experiment.

(2) The $\lambda_{k}=1$ approximation was proved to be systematically worse than the full calculation that includes the energy dependence of the $\lambda_{k}$ parameters. This assumption should be avoided in precise $\beta$ and $v$ spectra evaluations. 
(3) For the first time, the relevance of the $\xi$ approximation was thoroughly studied for many transitions. About half of the tabulated first forbidden nonunique transitions were reproduced well, but significant disagreement was found in all other cases. No specific tendency arose from the analysis of the present results other than highlighting that the condition $2 \xi / E_{0} \gg 1$ is not sufficient in itself. A conservative limit of the $2 \xi / E_{0}$ ratio of 100 was deduced, but has to be confirmed experimentally.

This study demonstrates also that the mean energy of a $\beta$ spectrum given in the nuclear databases is definitely erroneous for forbidden transitions. And even for an allowed transition, this mean energy is not trustworthy in the case of low $E_{0}$ because of the major influence of atomic effects. A full calculation is thus recommended for each data evaluation.
However, improving nuclear databases, e.g., with a recommended spectrum or an experimental shape factor, will not be helpful if this information is not systematically taken into account. Presently, most research communities and the nuclear power industry use their own codes to calculate the mean energies from the transition parameters given in the databases. These codes are based on the same assumptions which have been tested in the present work and thus an effort should be made to upgrade them. Furthermore, in the context of reactor $v$ oscillations, a strong impact can be expected on the method used to convert measured total fission $\beta$ spectra to total $v$ spectra. This impact should, at least, be estimated with improved calculations.

\section{ACKNOWLEDGMENTS}

This study was produced under the auspices and scientific support of the Decay Data Evaluation Project (DDEP) international collaboration.
[1] R. G. Helmer, E. Browne, and M.-M. Bé, J. Nucl. Sci. Tech. 39, 455 (2002).

[2] M. R. Bhat, in Evaluated Nuclear Structure Data File $(E N S D F)$, edited by S. M. Qaim, Nucl. Data Sci. Tech. (Springer-Verlag, Berlin, 1992), p. 817; Data extracted using the NNDC On-Line Data Service from the ENSDF database, files revised as of February 6, 2015.

[3] Computer code LOGFT Program, http://www.nndc.bnl.gov/ nndcscr/ensdf_pgm/analysis/logft/ (2001), original code from [24].

[4] T. W. Burrows, The Program RADLST, Tech. Rep. BNL-NSC52142 (Brookhaven National Laboratory, Upton, 1988).

[5] P. Huber, Phys. Rev. C 84, 024617 (2011).

[6] P. Huber, Phys. Rev. C 85, 029901(E) (2012).

[7] R. Broda, P. Cassette, and K. Kossert, Metrologia 44, S36 (2007).

[8] M. Bardiès and J.-F. Chatal, Phys. Med. Biol. 39, 961 (1994).

[9] A. L. Nichols, Summary Report of Consultants' Meeting on Beta-Decay and Decay Heat, Vienna, 12-14 December 2005, Tech. Rep. INDC (NDS)-0483 (International Nuclear Data Committee, IAEA Nuclear Data Section, Vienna, 2006).

[10] J. dos Anjos, A. Bernstein, N. Bowden, M. Cribier, E. Kemp, T. Lasserre, D. Lhuiller, R. de Meijer, T. Moriarty, D. Reyna, A. Starostin, F. Suekane, and J. Xiao, Final Report: Focused Workshop on Antineutrino Detection for Safeguards Applications, Vienna, 28-30 October 2008, Tech. Rep. STR361 (International Atomic Energy Agency, Vienna, 2009).

[11] H. V. Klapdor, Fortschr. Phys. 33, 1 (1985).

[12] M. Cribier, M. Fechner, T. Lasserre, A. Letourneau, D. Lhuillier, G. Mention, D. Franco, V. Kornoukhov, and S. Schönert, Phys. Rev. Lett. 107, 201801 (2011).

[13] A. C. Hayes, J. L. Friar, G. T. Garvey, G. Jungman, and G. Jonkmans, Phys. Rev. Lett. 112, 202501 (2014).

[14] X. Mougeot and C. Bisch, Phys. Rev. A 90, 012501 (2014).

[15] H. Behrens and W. Bühring, Electron Radial Wave Functions and Nuclear Beta Decay (Clarendon, Oxford, 1982).

[16] P. J. Mohr, B. N. Taylor, and D. B. Newell, Rev. Mod. Phys. 84, 1527 (2012).
[17] H. Behrens and J. Jänecke, in Numerical Tables for BetaDecay and Electron Capture, edited by H. Schopper, LandoltBörnstein, New Series, Group I, Vol. 4 (Springer Verlag, Berlin, 1969).

[18] M. E. Rose, Relativistic Electron Theory (Wiley, New York, 1961).

[19] W. Bühring, Nucl. Phys. 61, 110 (1965).

[20] H. F. Schopper, in Weak Interactions and Nuclear Beta Decay (North-Holland, Amsterdam, 1966), p. 284.

[21] J. W. Cooper and T. Åberg, Nucl. Phys. A 298, 239 (1978).

[22] M. E. Rose, Phys. Rev. 49, 727 (1936).

[23] L. T. Dillman, EDISTR - A Computer Program to Obtain a Nuclear Decay Data Base for Radiation Dosimetry, Tech. Rep. ORNL/TM-6689 (Oak Ridge National Laboratory, Oak Ridge, 1980).

[24] N. B. Gove and M. J. Martin, Nucl. Data Tables A 10, 205 (1971).

[25] A. Sirlin, Phys. Rev. 164, 1767 (1967).

[26] W. Jaus, Phys. Lett. 40, 616 (1972).

[27] A. Sirlin, Phys. Rev. D 84, 014021 (2011).

[28] H. Behrens and L. Szybisz, Shapes of beta Spectra, Physik Daten - Physics Data, Vol. 6-1 (Zentralstelle für Atomkernenergie-Dokumentation, EggensteinLeopoldshafen, 1976).

[29] Recommended Atomic and Nuclear Data Evaluated by the DDEP Collaboration, http://www.nucleide.org/DDEP_WG/ DDEPdata.htm (2015); Data extracted from the Nucléide database, files revised as of February 6, 2015.

[30] I. S. Towner and J. C. Hardy, Phys. Rev. C 72, 055501 (2005).

[31] J. W. F. Piel, Nucl. Phys. A 203, 369 (1973).

[32] A. Z. Schwarzschild, Ph.D thesis, Columbia University, 1957.

[33] Y. K. Lee, L. W. Mo, and C. S. Wu, Phys. Rev. Lett. 10, 253 (1963).

[34] C. S. Wu, Rev. Mod. Phys. 36, 618 (1964).

[35] H. Behrens, M. Kobelt, L. Szybisz, and W. G. Thies, Nucl. Phys. A 246, 317 (1975).

[36] V. V. Kuzminov and N. J. Osetrova, Yad. Fiz. 63, 1365 (2000) [Phys. Atomic Nuclei 63, 1292 (2000)]. 
[37] H. Daniel and U. Schmidt-Rohr, Nucl. Phys. 7, 516 (1958).

[38] I. Hofmann, Acta Phys. Austriaca 18, 309 (1964).

[39] D. W. Hetherington, A. Alousi, and R. B. Moore, Nucl. Phys. A 494, 1 (1989).

[40] H. J. Gils, D. Flothmann, R. Lohken, and W. Wiesner, Nucl. Instrum. Methods 105, 179 (1972).

[41] H. Genz, J. Reisberg, A. Richter, B. M. Schmitz, G. Schrieder, K. Werner, and H. Behrens, Nucl. Instrum. Methods 134, 309 (1976).

[42] B. M. Schmitz, Ph.D thesis, Bochum University, Bochum, 1976.

[43] W. Wiesner, D. Flothmann, and H. J. Gils, Nucl. Instrum. Methods 112, 449 (1973).

[44] T. Kruger, H. Appel, W. G. Thies, and H. Behrens, Phys. Lett. B 121, 303 (1983).

[45] H. Paul, Acta Phys. Austriaca 18, 315 (1964).

[46] H. J. Fischbeck, Phys. Rev. 173, 1078 (1968).

[47] D. Flothmann, W. Wiesner, R. Lohken, and H. Rebel, Z. Phys. 225, 164 (1969).

[48] H. E. Bosch, M. Behar, M. C. Cambiaggio, G. Garcia Bermudez, and L. Szybisz, Can. J. Phys. 51, 2260 (1973).

[49] J. H. Hamilton, L. M. Langer, and D. R. Smith, Phys. Rev. 123, 189 (1961).

[50] J. I. Rhode and O. E. Johnson, Phys. Rev. 131, 1227 (1963).

[51] K. S. R. Sastry, Tech. Rep. AD-752621 (University of Massachusetts, Amherst, 1972).

[52] C. Le-Bret, Ph.D thesis, Université Paris Sud - Paris XI, 2012.

[53] D. C. Camp and L. M. Langer, Phys. Rev. 129, 1782 (1963).

[54] W. F. Slot, G. H. Dulfer, H. van der Molen, and H. Verheul, Nucl. Phys. A 186, 28 (1972).

[55] J. H. Hamilton, L. M. Langer, and W. G. Smith, Phys. Rev. 119, 772 (1960).

[56] H. Daniel, O. Mehling, and D. Schotte, Z. Phys. 172, 202 (1963).

[57] H. E. Bosch, J. Davidson, M. A. Fariolli, and V. Silbergleit, Nucl. Instrum. Methods 117, 213 (1974).

[58] H. Daniel, G. T. Kaschl, H. Schmitt, and K. Springer, Phys. Rev. 136, B1240 (1964).

[59] H. Daniel, M. Kuntze, B. Martin, P. Schmidlin, and H. Schmitt, Nucl. Phys. 63, 145 (1965).

[60] H. Daniel, O. Mehling, P. Schmidlin, D. Schotte, and E. Thummernicht, Z. Phys. 179, 62 (1964).

[61] S. T. Hsue, M. U. Kim, L. M. Langer, and E. H. Spejewski, Nucl. Phys. A 109, 423 (1968).

[62] H. Beekhuis, Phys. Lett. 21, 205 (1966).

[63] F. G. A. Quarati, I. V. Khodyuk, C. W. E. van Eijk, P. Quarati, and P. Dorenbos, Nucl. Instrum. Methods Phys. Res., Sect. A 683, 46 (2012).

[64] J. van Klinken, F. Pleiter, and H. T. Dijkstra, Nucl. Phys. A 112, 372 (1968).

[65] G. R. Kartashov, N. A. Burgov, and A. V. Davydov, Izv. Akad. Nauk SSSR, Ser. Fiz. 25, 189 (1961) [Columbia Tech. Transl. 25, 184 (1962)].

[66] H. M. W. Booij, E. A. van Hoek, H. van der Molen, W. F. Slot, and J. Blok, Nucl. Phys. A 160, 337 (1971).

[67] T. Nagarajan, M. Ravindranath, and K. V. Reddy, Nucl. Phys. A 137, 467 (1969).

[68] C. N. Rao, B. M. Rao, P. M. Rao, and K. Venkata Reddy, Phys. Rev. C 11, 1735 (1975).
[69] F. K. Wohn, W. L. Talbert, and W. L. Talbert, Nucl. Phys. A 146, 33 (1970).

[70] J. I. Rhode, O. E. Johnson, and W. G. Smith, Phys. Rev. 129, 815 (1963).

[71] D. Flothmann, H. J. Gils, W. Wiesner, and R. Lohken, Z. Phys. 272, 219 (1975).

[72] D. Flothmann, Ph.D thesis, Heidelberg University, 1972.

[73] H. E. Bosch, J. Davidson, V. Silbergleit, C. A. Heras, and S. M. Abecasis, Z. Phys. 273, 373 (1975).

[74] S. Andre, P. Liaud, F. Perales, and S. Y. Van der Werf, Phys. Lett. B 30, 160 (1969).

[75] P. G. Hansen, H. L. Nielsen, K. Wilsky, Y. K. Agarwal, C. V. K. Baba, and S. K. Bhattacherjee, Nucl. Phys. 76, 257 (1966).

[76] E. Huster and H. Verbeek, Z. Phys. 203, 435 (1967).

[77] L. G. Elliott, M. A. Preston, and J. L. Wolfson, Can. J. Phys. 32, 153 (1954).

[78] L. G. Elliott, M. A. Preston, and J. L. Wolfson, Can. J. Phys. 33, 607 (1955).

[79] L. Feldman and C. S. Wu, Phys. Rev. 87, 1091 (1952).

[80] B. T. Wright, Phys. Rev. 90, 159 (1953).

[81] J. L. Wolfson, Can. J. Phys. 34, 256 (1956).

[82] H. Leutz, G. Schulz, and H. Wenninger, Z. Phys. 187, 151 (1965).

[83] C. Bisch, Ph.D thesis, Université de Strasbourg, CEA-R-6393, 2014.

[84] B. Mallikarjuna Rao, C. Narasimha Rao, P. Mallikharjuna Rao, R. Mathews, and K. Venkata Reddy, Curr. Sci. (India) 45, 319 (1976).

[85] R. D. Connor, T. J. Goldman, and I. L. Fairweather, Proc. Roy. Soc. Edinburgh, Sect. A 70, 67 (1972).

[86] L. M. Langer and D. R. Smith, Phys. Rev. 119, 1308 (1960).

[87] H. Daniel, W. Collin, M. Kuntze, S. Margulies, B. Martin, O. Mehling, P. Schmidlin, and H. Schmitt, Nucl. Phys. A 118, 689 (1968).

[88] O. E. Johnson and W. G. Smith, Phys. Rev. 118, 1315 (1960).

[89] T. Nagarajan, M. Ravindranath, and K. V. Reddy, Phys. Rev. C 3, 247 (1971).

[90] T. Nagarajan, M. Ravindranath, and K. V. Reddy, Phys. Rev. C 3, 254 (1971).

[91] R. P. Sharma and H. G. Devare, Phys. Rev. 131, 384 (1963).

[92] S. T. Hsue, M. U. Kim, L. M. Langer, J. W. F. Piel, and E. J. Spejewski, Nucl. Phys. A 117, 686 (1968).

[93] T. Nagarajan, M. Ravindranath, and K. V. Reddy, Nucl. Phys. A 134, 433 (1969).

[94] T. Nagarajan, M. Ravindranath, and K. Venkata Reddy, Nuovo Cimento A 8, 305 (1972).

[95] H. Beekhuis and R. J. Van Duinen, Nucl. Phys. A 108, 382 (1968).

[96] H. Daniel and G. T. Kaschl, Nucl. Phys. 76, 97 (1966).

[97] R. Hess, P. Lipnik, C. F. Perdrisat, and J. W. Sunier, Nucl. Phys. 54, 673 (1964).

[98] B. Persson and J. Reynolds, Nucl. Phys. 66, 439 (1965).

[99] H. Beekhuis, P. Boskma, J. Van Klinken, and H. De Waard, Nucl. Phys. 79, 220 (1966).

[100] T. Nagarajan, M. Ravindranath, and K. V. Reddy, Nuovo Cimento A 3, 689 (1971).

[101] H. M. Schupferling and K. W. Hoffmann, Z. Phys. 266, 129 (1974).

[102] Y. Yoshizawa, H. Okamura, S. Iwata, I. Fujiwara, T. Shigematsu, M. Tabushi, T. Tarumoto, and K. Sakamoto, Nucl. Phys. 46, 78 (1963). 
[103] P. G. Hansen, H. L. Nielsen, and K. Wilsky, Nucl. Phys. 89, 571 (1966).

[104] S. Y. van der Werf, H. de Waard, and H. Beekhuis, Nucl. Phys. A 134, 215 (1969).

[105] P. M. Rao, B. M. Rao, C. N. Rao, R. Mathews, and K. V. Reddy, Curr. Sci. (India) 44, 755 (1975).

[106] S. Y. van der Werf, Z. Phys. 259, 45 (1973).

[107] E. H. Spejewski, Nucl. Phys. 82, 481 (1966).

[108] T. Nagarajan, M. Ravindranath, K. V. Reddy, and S. Jnanananda, Phys. Rev. 178, 1968 (1969).

[109] J. P. Deutsch, L. Grenacs, and J. Lehmann, in Kolloquium über Beta-Zerfall und Schwache Wechselwirkungen (Max Planck Institut für Kernphysik, Heidelberg, 1965), p. 257.

[110] H. Paul, Nucl. Phys. 72, 326 (1965).

[111] W. Wiesner, D. Flothmann, H. J. Gils, R. Lohken, and H. Rebel, Nucl. Phys. A 191, 166 (1972).

[112] D. Flothmann, R. Lohken, W. Wiesner, and H. Rebel, Phys. Rev. Lett. 25, 1719 (1970).

[113] J. M. Trischuk and E. Kankeleit, Nucl. Phys. A 90, 33 (1967).

[114] H. Behrens, M. Kobelt, W. G. Thies, and H. Appel, Z. Phys. 252, 349 (1972).

[115] A. Grau Carles, Nucl. Instrum. Methods Phys. Res., Sect. A 551, 312 (2005).
[116] K. Kossert, O. J. Nahle, and A. Grau Carles, Appl. Radiat. Isot. 69, 1246 (2011).

[117] H. Rotzinger, M. Linck, A. Burck, M. Rodrigues, M. Loidl, E. Leblanc, L. Fleischmann, A. Fleischmann, and C. Enss, J. Low Temp. Phys. 151, 1087 (2008).

[118] S. Raman, H. Kawakami, S. Ohya, and Z. Matumoto, Phys. Rev. C 9, 2463 (1974).

[119] H. Daniel, Z. Phys. 150, 144 (1958).

[120] J. C. Hocquenghem, S. Andre, and P. Liaud, J. Phys. (Paris) 29, 138 (1968).

[121] M. Reich and H. M. Schupferling, Z. Phys. 271, 107 (1974).

[122] E. der Mateosian and C. S. Wu, Phys. Rev. 95, 458 (1954).

[123] P. Lidofsky, E. Alperovitch, and C. S. Wu, Phys. Rev. 90, 387A (1953).

[124] H. Behrens and P. Christmas, Nucl. Phys. A 399, 131 (1983).

[125] A. Grau Carles and K. Kossert, Nucl. Instrum. Methods Phys. Res., Sect. A 572, 760 (2007).

[126] P. Belli, R. Bernabei, N. Bukilic, F. Cappella, R. Cerulli, C. J. Dai, F. A. Danevich, J. R. de Laeter, A. Incicchitti, V. V. Kobychev, S. S. Nagorny, S. Nisi, F. Nozzoli, D. V. Poda, D. Prosperi, V. I. Tretyak, and S. S. Yurchenko, Phys. Rev. C 76, 064603 (2007). 


\section{Erratum: Reliability of usual assumptions in the calculation of $\beta$ and $v$ spectra [Phys. Rev. C 91, 055504 (2015)]}

Unfortunately, a typographical error is present in Eq. (13) of Sec. II E for the radiative corrections. Originally, the third line of Eq. (13) which defines the quantity $g(W, q)$ was given as

$$
\cdots+\frac{\tanh ^{-1} \beta}{\beta}\left[\left(1+\beta^{2}\right) \frac{q^{2}}{3 W^{2}}-4 \tanh ^{-1} \beta\right],
$$

but should be

$$
\cdots+\frac{\tanh ^{-1} \beta}{\beta}\left[2\left(1+\beta^{2}\right)+\frac{q^{2}}{6 W^{2}}-4 \tanh ^{-1} \beta\right]
$$

see Refs. [15,25].

Note that the correct formula was used throughout the work, and therefore all results given previously remain valid.

[15] H. Behrens and W. Bühring, Electron Radial Wave Functions and Nuclear Beta Decay (Clarendon, Oxford, 1982).

[25] A. Sirlin, Phys. Rev. 164, 1767 (1967). 